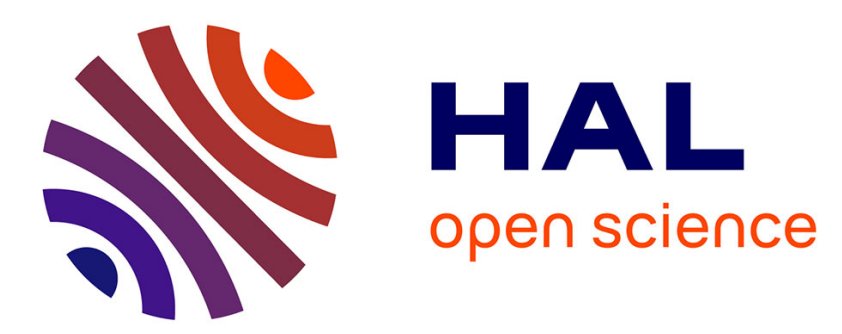

\title{
Hybrid pulse propagation model and quasi-phase-matched four-wave mixing in multipass cells
}

Marc Hanna, Nour Daher, Florent Guichard, Xavier Delen, Patrick Georges

\section{To cite this version:}

Marc Hanna, Nour Daher, Florent Guichard, Xavier Delen, Patrick Georges. Hybrid pulse propagation model and quasi-phase-matched four-wave mixing in multipass cells. Journal of the Optical Society of America B, 2020, 37 (10), pp.2982. 10.1364/JOSAB.395789 . hal-02941634

\section{HAL Id: hal-02941634 \\ https://hal-iogs.archives-ouvertes.fr/hal-02941634}

Submitted on 17 Sep 2020

HAL is a multi-disciplinary open access archive for the deposit and dissemination of scientific research documents, whether they are published or not. The documents may come from teaching and research institutions in France or abroad, or from public or private research centers.
L'archive ouverte pluridisciplinaire HAL, est destinée au dépôt et à la diffusion de documents scientifiques de niveau recherche, publiés ou non, émanant des établissements d'enseignement et de recherche français ou étrangers, des laboratoires publics ou privés. 


\title{
Hybrid pulse propagation model and quasi- phase matched four-wave mixing in multipass cells
}

\author{
Marc Hanna, ${ }^{1},{ }^{*}$ Nour Daher, ${ }^{1}$ Florent Guichard,${ }^{2}$ Xavier DÉlen,,${ }^{1}$ \\ AND PATRICK GEORGES ${ }^{1}$ \\ ${ }^{1}$ Université Paris-Saclay, Institut d'Optique Graduate School, CNRS, Laboratoire Charles Fabry, \\ 91127, Palaiseau, France. \\ ${ }^{2}$ Amplitude Laser, 11 Avenue de Canteranne, Cité de la Photonique, 33600 Pessac, France. \\ *marc.hanna@institutoptique.fr
}

\begin{abstract}
We describe a nonlinear propagation model based on a generalized Schrödinger equation in the time domain coupled to Gaussian beam evolution through ABCD matrices that account for Kerr lensing in the spatial domain. This model is well suited to simulate propagation in mildly nonlinear systems such as multipass cells for temporal compression. It is validated against both a full $(x, y, z, t)$ numerical model and recently reported experimental results in multipass cells, with excellent agreement. It also allows us to identify the physical mechanism for the recently reported parasitic appearance of spectral content in the $700-950 \mathrm{~nm}$ range in argon-filled multipass cells that are used to compress pulses at $1030 \mathrm{~nm}$. We think this is due to a quasi-phase matched degenerate four-wave mixing process. This process could be used in the future to perform wavelength conversion as is already done in fibers and capillaries.
\end{abstract}

(C) 2020 Optical Society of America

\section{Introduction}

Pulse propagation models have been routinely used for decades both to design experiments and interpret their results in the field of ultrafast photonics [1,2]. They can include physical phenomena such as dispersion, diffraction, loss, gain, and nonlinearity, with models spanning a wide range of complexity, and taking into account a number of dimensions going from $2 \mathrm{D}$ $(t, z)$ or $(x, z)$ to $4 \mathrm{D}(x, y, z, t)$. The value of these models is given by their ability to predict or reproduce the experimental observations in a qualitative or quantitative manner, and to do so in a reasonable computing time. This requires adapting the model complexity to the physics at hand.

As an example, nonlinear propagation in single transverse mode fibers can be very well described with $(t, z)$ models, assuming that the transverse field distribution is the fundamental mode of the fiber. Although this is an approximation at high nonlinearity level, when the spatial Kerr effect starts modifying the transverse index distribution locally and instantaneously, a very large number of studies have successfully relied on these models to interpret the obtained data and design experiments [3].

Modelling highly nonlinear ultrafast phenomena in freespace such as filamentation [4] or supercontinuum generation [5], on the other hand, requires at least an additional space dimension if a cylindrical symmetry is assumed, or a full 4D model. This allows an accurate description of space-time couplings that are almost always observed in experiments. In addition, strong field light-matter interaction effects such as ionization and its impact on propagation, or higher-order nonlinearity, must be accounted for since they play a central role in this physics. These models are complex and require a much larger computing effort than the $(t, z)$ models used for singlemode fiber, which somehow restricts their use and value.

In this article, we describe a nonlinear propagation model in freespace that is adapted to situations where (i) the impact of nonlinearity on the spatial profile is reduced to a change in size and radius of curvature, which allows to describe it as a Gaussian beam and (ii) negligible 
space-time couplings are introduced on the propagating field. It is based on a split-step method that propagates the field in time using a generalized nonlinear Schrödinger equation and in space using an ABCD matrix model that accounts for diffraction and Kerr lens. The time and space parts are coupled through the evolution of peak intensity and beam size. The model presented here is particularly well suited to describe propagation in nonlinear regenerative amplifiers [6], or MultiPass Cells (MPCs) [7-10], where it was shown that an injected Gaussian beam essentially retains its character upon propagation and that space-time couplings are only observed at extreme levels of nonlinearity [11,12]. Compared to the model presented in [11], that is purely $(t, z)$ without a description of spatial effects, the model presented here allows to take into account Kerr lensing that can have non negligible impact on the beam caustic, as will be discussed hereunder. Compared to full 4D dimensional models [13], it is much faster in terms of computing time, and allows to account for the impact of beam imperfections on the level of nonlinearity easily through an $\mathrm{M}^{2}$ beam quality factor. Although several models using the Gaussian ansatz in both space and time have already been proposed [14,15], they fail to predict the complex temporal behavior induced by nonlinear propagation, leading to pulses that cannot be described by a Gaussian ansatz in the time domain, and are therefore not appropriate in the MPC context.

The article is organized as follows. In the first part, we first describe the temporal and spatial parts of the model and how they are coupled. We compare its results both to a full 4D model and to the very simple 2D model described in [11], showing that the level of physical description adopted is ideal for the particular problem of propagation in MPCs. In a second part, we apply the model to reproduce the experimental data obtained in high-energy gas-filled MPC experiments that use near concentric configurations [16,17]. In this case, the small beam size and large divergence at the MPC waist makes 4D simulations particularly computationally intensive, underlining the usefulness of our model. The experimental data is quantitatively reproduced, and the numerical results allow us to interpret an observation reported in both cases: the generation of spectral content in the $700-950 \mathrm{~nm}$ range. We believe it is due to quasi-phase-matched (QPM) four-wave mixing (FWM) in normal dispersion regime, and provide the corresponding phase matching curve to back this interpretation.

The model can easily describe propagation in both gas-filled MPCs and MPCs including plates of nonlinear material, include dispersion imparted by both material and mirror coatings, and all third-order nonlinear effects such as self-phase modulation, self-steepening, and Raman scattering. It provides an efficient tool for the design and data analysis of these nonlinear subsystems that are currently being developed at a fast pace.

\section{Model description and validation}

\subsection{Description}

Propagation in the time domain is modeled using the following generalized Schrödinger equation [1]:

$$
\frac{\partial A}{\partial z}=-\left(i \frac{\beta_{2}}{2} \frac{\partial^{2}}{\partial t^{2}}-\frac{\beta_{3}}{6} \frac{\partial^{3}}{\partial t^{3}}+\cdots\right) A+i \gamma\left(1+\frac{i}{\omega_{0}} \frac{\partial}{\partial t}\right) A \int_{0}^{\infty} R\left(t^{\prime}\right)\left|A\left(z, t-t^{\prime}\right)\right|^{2} d t^{\prime},(1)
$$

where $A(z, t)$ is the field normalized so that $|A(z, t)|^{2}$ is the optical power, $z$ is the propagation distance, $t$ is time, $\beta_{n}$ is the $\mathrm{n}$-th order Taylor coefficient of the propagation constant in the material $\beta(\omega)=\omega n(\omega) / c, \omega_{0}$ is the central angular frequency, $c$ is the speed of light in vacuum, and $R(t)$ is the normalized third-order nonlinear response of the material that includes the instantaneous electronic contribution and delayed molecular contributions. Since the dispersive part of this propagation equation is implemented in the frequency domain using a split-step method [1], the full spectral dependence of the local index of refraction $n(\omega)$ is taken into account using an appropriate Sellmeier equation. Quantum noise can be added using a one photon per mode approach [3]. This temporal equation is coupled to the spatial evolution 
through the nonlinear coefficient $\gamma=n_{2} \omega_{0} / c A_{\text {eff }}$, where $n_{2}$ is the nonlinear index of the material and $A_{\text {eff }}=\pi w(z)^{2}$ is the effective area of the beam. The beam radius $w$ evolves according to the spatial part of the propagation model described below.

The linear and nonlinear material properties are changed as a function distance to account for propagation in the various materials of the MPC, and discrete transfer functions can be added to account for the spectral reflectivity and dispersion of the coatings on mirrors or bulk plates.

The spatial part of the model consists in propagating the reduced complex Gaussian parameter $\hat{q}$ using the same step $d z$ as in the split-step algorithm used to solve the temporal propagation equation. This parameter is defined as

$$
\frac{1}{\hat{q}}=\frac{1}{\hat{R}}+i \frac{\lambda_{0}}{\pi w^{2}},
$$

where $1 / \widehat{R}=n / R$ is the reduced radius of curvature and $\lambda_{0}$ is the central wavelength in vacuum. This parameter completely determines the Gaussian spatial profile and is propagated according to the usual ABCD matrix formalism [18] according to

$$
\hat{q}_{z+d z}=\frac{A \hat{q}_{z}+B}{C \hat{q}_{z}+D}
$$

The $\mathrm{ABCD}$ matrix that describes propagation over a length $d z$ of material is given by

$$
M=\left(\begin{array}{cc}
1-\frac{d z}{2 n f_{\text {Kerr }}} & \frac{d z}{n}\left(1-\frac{d z}{4 f_{\text {Kerr }}}\right) \\
-\frac{1}{f_{\text {Kerr }}} & 1-\frac{d z}{2 n f_{\text {Kerr }}}
\end{array}\right) .
$$

It corresponds to propagation in freespace in a material with index of refraction $n$ over a length $d z$, in which a lens with focal length $f_{\text {Kerr }}$ is introduced at half distance. Because of the dependence of the $\mathrm{ABCD}$ matrix on the beam size, the model must be implemented by incrementing the distance by small steps $d z$ over which the beam size and radius of curvature change only infinitesimally. The definition of the nonlinear focal length is not trivial, and leads to different results depending on the model used for propagation [19]. The approach we take to evaluate a physically meaningful value is as follows. We start from the precise expression established for the critical power for a Gaussian beam $[2,20] P_{\text {crit }}=3.77 \frac{\lambda_{0}^{2}}{8 \pi n n_{2}}$. This expression corresponds to exact compensation between diffraction and self-focusing, although it is an unstable physical situation. Nonlinear propagation of a time-independent Gaussian beam of power $P_{\text {peak }}$ using the model described above is then simulated, with a Kerr focal length equal to

$$
f_{\text {Kerr }}=K \frac{\pi w^{4}}{P_{\text {peak }} n_{2} d z},
$$

where $K$ is a constant that can be adjusted. Apart from the constant, this expression corresponds to the expression of the Kerr focal length obtained by using a parabolic approximation for the Gaussian beam intensity profile [21]. The value of $\mathrm{K}$ is then adjusted to obtain catastrophic self-focusing for Ppeak> Pcrit and diffraction for Ppeak<Pcrit upon freespace propagation in the nonlinear material. We find that this corresponds to $\mathrm{K}=0.47$. When coupled to the time domain model, the value of Kerr focal length is defined using the peak power, although one could argue that this focal length varies instantaneously. The nature of our model imposes the 
absence of space-time couplings, and the peak power is therefore used since it corresponds to the maximum energy density in the time domain.

Imperfections in the beam can easily be taken into account in a first approximation by using an $M^{2}$ factor. In this case, an equivalent beam size of $w \times M$ must be used when evaluating the nonlinear parameter $\gamma$ and the nonlinear focal length $f_{K e r r}$. In addition, by keeping track of two different $\hat{q}$ parameters for both transverse dimensions, the propagation of elliptical and/or astigmatic Gaussian beams can easily be accounted for.

Possible ionization of the medium is not taken into account in this model. This phenomenon is very threshold-like and its onset quickly leads to drastic modifications of the beam shape. We therefore considered that it was not relevant in a model that assumes a Gaussian beam shape. The maximum intensity must therefore be monitored to ensure that the ionization rate is negligible.

The overall model is implemented using a propagation step $d z$ small enough to guarantee small changes in beam size, radius of curvature, temporal and spectral profiles. The temporal model is coupled to the spatial part through the value of peak power, which is coupled back to the time domain through the value of the beam size that defines the level of nonlinearity $\gamma$. Note that although this model is valid for spatial profiles close to a Gaussian beam, it is quite general in terms of temporal / spectral intensity and phase profiles. We now examine the validity of this model by comparing it to other approaches to compute the propagation of pulses in a test case.

\subsection{Validation}

The test case we choose is the same as in our previous work [11], to allow direct comparison between three numerical models. The first model is a 4D model described in [13], that fully accounts for diffraction, dispersion and third order nonlinearity by solving an envelope equation [22] using the split-step method in space and time. The second model is a purely 2D model that assumes a constant beam size. This beam size is calculated by averaging the nonlinearity accumulated by the linear stationary beam in the MPC over one roundtrip, as described in [11]. The third model is the one described in this article.

We model propagation in a near concentric MPC composed of two mirrors with radius of curvature $300 \mathrm{~mm}$ separated by $551 \mathrm{~mm}$, filled with 3 bars of xenon. The input pulses are Gaussian in space and time with a full width at half maximum (FWHM) pulse duration of 350 $\mathrm{fs}$, an energy of $200 \mu \mathrm{J}$, and a central wavelength of $1030 \mathrm{~nm}$. The corresponding spectral FWHM is $4.5 \mathrm{~nm}$. They are injected in the cell with a spatial Gaussian parameter that corresponds to the linear stationary beam and are propagated over 5 roundtrips. The numerical simulation parameters are the following. For the 4D model, the grid steps are $d x=d y=60 \mu \mathrm{m}$, $d z=5 \mathrm{~mm}, d t=20 \mathrm{fs}$, and the grid size is $6 \mathrm{~mm} \times 6 \mathrm{~mm}$ in the transverse plane and $2 \mathrm{ps}$ in the time domain. For the constant beam size model and the hybrid model, the temporal grid is the same, and the longitudinal step size is $d z=1 \mathrm{~mm}$. The results are plotted in Fig. 1. 

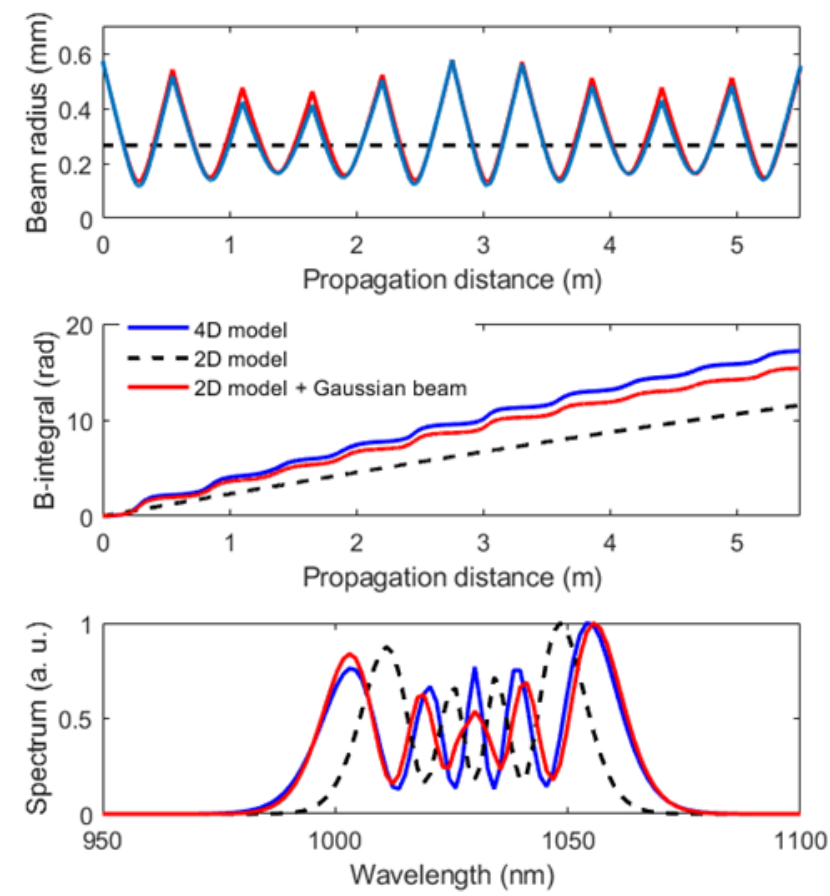

Fig. 1. Comparison of the three numerical models. Top - Evolution of the beam caustic. Middle - Accumulated B-integral as a function of distance. Bottom - Output spectrum. The blue solid lines correspond to the 4D model, the black dashed line to a constant beam size 2D model, the red line to the hybrid model presented in this paper.

As already described in [11], these parameters correspond to a high level of nonlinearity $\left(P_{\text {peak }} / P_{\text {crit }}=0.6\right)$, for which the simplified spatially averaged approach significantly underestimates the level of nonlinearity, resulting in a lower amount of spectral broadening. The reason for this is that nonlinearity in the space domain leads to significant changes in the beam size upon propagation, as can be observed in Fig. 1 (top), which is not taken into account by the simple constant beam size approach of the 2D model.

In contrast, by coupling nonlinear beam size evolution to the 2D model as described in this article, we are able to faithfully reproduce the caustic evolution, B-integral accumulation, and output spectrum as clearly observed in Fig. 1. There remains a small underestimation of the nonlinearity, but it has no significant impact on the output spectrum. This could be caused by a slight spatial intensity profile deviation from a purely Gaussian beam. To conclude this part, the simple model presented here allows us to describe highly nonlinear propagation in MPCs better than the longitudinally invariant model, and is relevant as long as no significant space time couplings or deviation from the Gaussian beam shape are induced. These hypotheses have been observed to hold for MPCs in almost all experimental reports. The added computational cost compared to a purely 2D model is negligible, with the benefit that the caustic evolution can be predicted. As shown above, this allows a more precise evaluation of the nonlinearity level, but it is also very useful to design experiments. Indeed, since nonlinear caustic evolution deviates significantly from the linear stationary beam, the maximum fluence on the mirrors or intensity in the gas are also changed, and this model allows for a more realistic prediction of mirror damage and gas ionization thresholds. We now use this model to compare its results to previous experimental reports.

\section{Application to near concentric gas-filled MPCs}




\subsection{Benchmarking against experimental reports}

We have chosen two reports that implement near concentric gas-filled MPC configurations, for which the computation effort of a $4 \mathrm{D}$ approach requires more than a common laptop computer. In contrast, the modified 2D model proposed here typically takes a few tens of seconds to run on such a machine. The experiments are described in [16,17]. The model is implemented without any adjustable parameters based on the information provided in these references. The most significant difference between the experiments and the data used in the model is that we have assumed an ideal Gaussian temporal profile at the input.
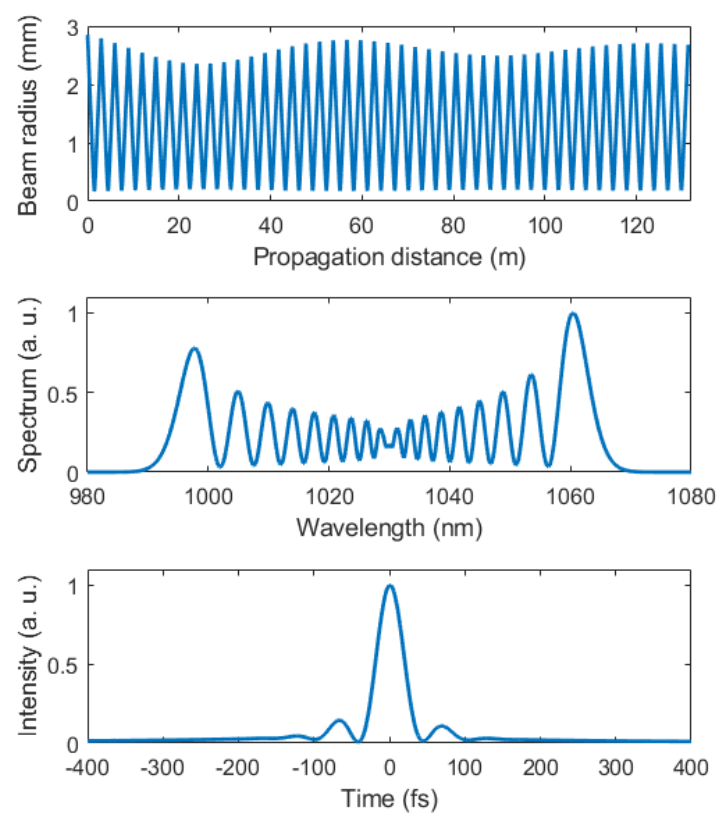

Fig. 2: Numerical results corresponding to the experiment reported in [16]. Top - Beam caustic evolution. Middle - Output spectrum. Bottom - Output temporal profile of the compressed pulse.

The first experiment that we numerically reproduce is described in [16], and corresponds to the current record in terms of pulse energy compressed using an MPC. Input pulses of $18 \mathrm{~mJ}$ at $5 \mathrm{kHz}$ repetition rate are compressed from $1.3 \mathrm{ps}$ down to $40 \mathrm{fs}$ in a $2.985 \mathrm{~m}$ long MPC with $1.5 \mathrm{~m}$ radius of curvature mirrors, filled with argon at 0.6 bar. The hybrid 2D model is implemented with a temporal step $d t=2 \mathrm{fs}$ on a temporal window of $10 \mathrm{ps}$, with a longitudinal spatial step $d z=5 \mathrm{~mm}$. The results of the simulation are shown in Fig. 2, and are in quantitative agreement with the reported experimental results. The amount of group delay dispersion (GDD) used to compress the pulses (by maximizing the peak power) in the simulation is $-7600 \mathrm{fs}^{2}$, as opposed to $-9400 \mathrm{fs}^{2}$ reported in [16]. This discrepancy might be due to dispersion of the MPC mirrors or other elements such as windows in the experiment, or possibly slight quadratic phase in the input beam. The simulated output pulse duration is $38 \mathrm{fs}$, in excellent agreement with the experiment, as well as the obtained output spectrum, that reproduces accurately the experimentally observed broadening and peak structure. The caustic evolution depicted in Fig. 2 (top) shows that the nonlinearity induces slow and relatively low amplitude oscillations on the beam size, in addition to the roundtrip modulation caused by propagation in the MPC. 

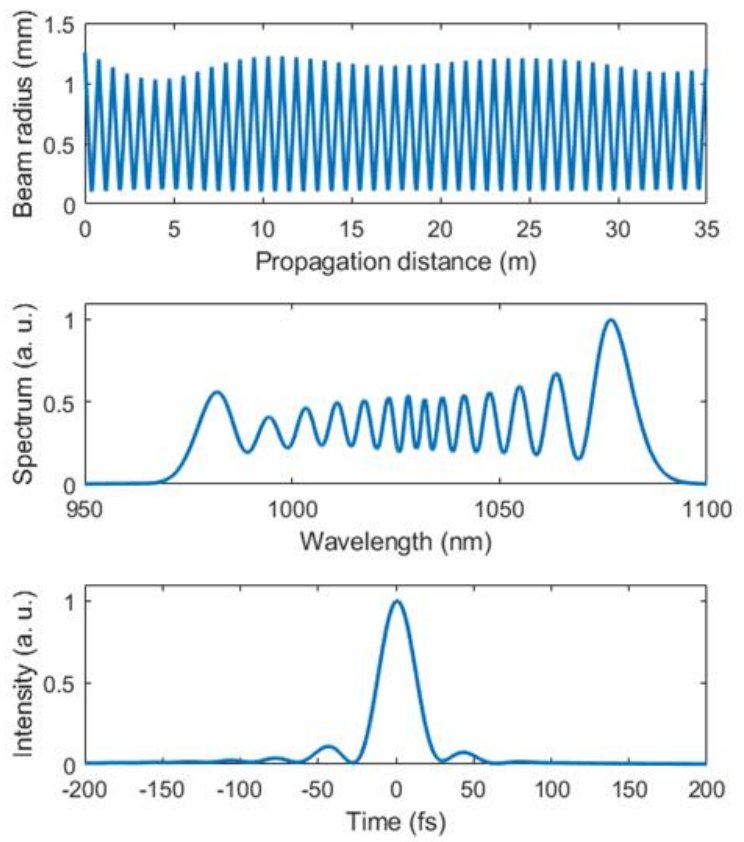

Fig. 3: Numerical results corresponding to the experiment reported in [17]. Top - Beam caustic evolution. Middle - Output spectrum. Bottom - Output temporal profile of the compressed pulse.

The second experiment we use to benchmark the model is reported in [17], and corresponds to the highest average power ever reported in an MPC-based nonlinear compression setup. Input pulses of $1.1 \mathrm{~mJ}$ at $500 \mathrm{kHz}$ repetition rate are compressed from 590 fs down to 30 fs in a $794 \mathrm{~mm}$-long MPC made of $400 \mathrm{~mm}$ radius of curvature mirrors filled with argon at 4 bar. The hybrid model is implemented with a temporal step $d t=2 \mathrm{fs}$ on a temporal window of 10 ps, with a longitudinal spatial step $d z=5 \mathrm{~mm}$. The simulation results are plotted in Fig. 3. The output pulses are compressed with a pure GDD of $-4400 \mathrm{fs}^{2}$, as opposed to $-5300 \mathrm{fs}^{2}$ in the experiment. The output pulse duration is $27 \mathrm{fs}$, in agreement with the experiment. One noticeable difference is the absence of a spectral peak at $1030 \mathrm{~nm}$ in the simulation, which is easily explained by the fact that a pure Gaussian input pulse in the time domain was used as the initial condition. The experimentally observed spectral peak is typically due to a residual pedestal of the input pulse in the time domain that is not spectrally broadened. Apart from this central peak at $1030 \mathrm{~nm}$, the simulated output spectrum reproduces well both the overall broadening and the peak structure observed in the experimental report. We now examine a feature reported by both reports in light of the numerical model.

\subsection{Quasi phase matched four-wave mixing in MPCs}

Both experimental demonstrations mentioned in the previous section report the generation of spectral content in the frequency range $700-950 \mathrm{~nm}$ at maximum power / nonlinearity. To investigate this observation, Fig. 4 shows the numerical output spectrum corresponding to reference [16] in the broad spectral range $800-1400 \mathrm{~nm}$ using a logarithmic scale, generated using the hybrid 2D model. We observe the appearance of spectral content around $860 \mathrm{~nm}$ and $1280 \mathrm{~nm}$, which is reminiscent of the signature of phase-matched degenerate FWM that can be observed e. g. in fibers $[23,24]$. In this phenomenon, two photons are annihilated at the pump wavelength while one signal and one idler photons are created on each spectral side. This process satisfies conservation of energy and momentum, and can be efficient if phase matching is satisfied. In the absence of contributions from waveguide dispersion, this requires that the 
pump wavelength lies in the anomalous dispersion region of the medium [1]. However, it has been shown that phase-matching can be achieved in fibers in the normal dispersion regime by exploiting waveguide dispersion $[25,26]$.

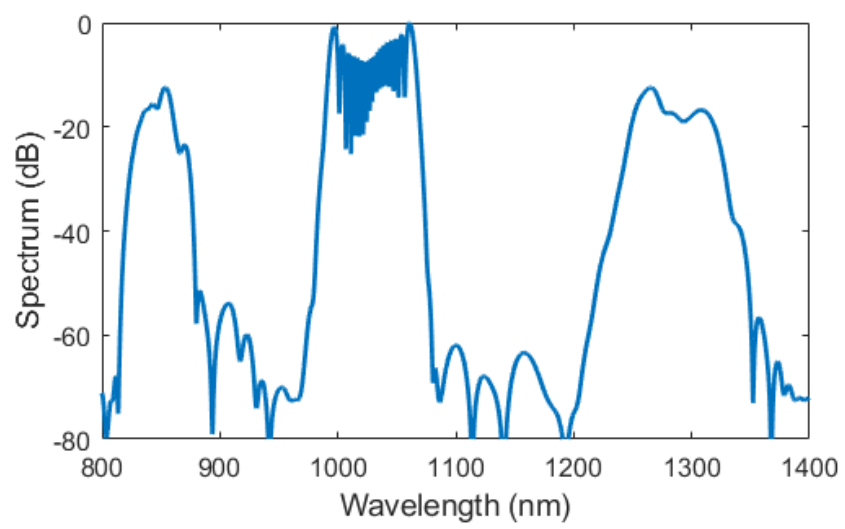

Fig. 4: Numerical output spectrum corresponding to reference [16] in logarithmic scale.

Since propagation in MPCs is in freespace, and because $1030 \mathrm{~nm}$ is located in the normal dispersion region for argon, there is no obvious possibility to attribute the reported observation to a phase-matched FWM process. However, both these observations were made in concentric MPCs, where the nonlinearity is highly located at the cell waist. The nonlinearity $\gamma(z)$ is therefore a periodic function with period $L_{M P C}$, defined as the distance between the mirror cells. This opens the possibility of a QPM process [27]: energy transfer to the sidebands occurs upon propagation through the waist (over the Rayleigh range in a first approximation), and quasi linear propagation in the remainder of the cell resynchronizes the relative idler, signal and pump phases for the energy transfer at the next waist.

To further investigate if the observation is compatible with this physical origin, we evaluate the corresponding phase matching condition in MPCs. The pump, signal, and idler angular frequencies must satisfy energy conservation $2 \omega_{p}=\omega_{s}+\omega_{i}$. Considering only the linear contribution to phase matching as a first simple approximation, the QPM condition can be written as

$$
\Delta \beta_{L}=\beta\left(\omega_{s}\right)+\beta\left(\omega_{i}\right)-2 \beta\left(\omega_{p}\right)=\frac{2 m \pi}{L_{M P C}},
$$

where $m$ is an integer. The propagation constants in Eq. (6) are simply determined by the gas nature and pressure, neglecting contributions from other sources such as mirror coatings. The QPM process can be more efficient if the region where nonlinearity occurs is shorter than half the coherence length $L_{c o h}=2 \pi /\left|\Delta \beta_{L}\right|$, so that the energy always transfers from the pump to the signal and idler. In the non-depleted pump and weak signal / idler approximation, the nonlinear contribution to phase matching can be readily included as $\Delta \beta_{N L}(z)=2 \gamma(z) P_{\text {peak }}$ [1]. In this case, the QPM condition becomes

$$
\int_{0}^{L_{M P C}}\left(\Delta \beta_{L}+\Delta \beta_{N L}\right) d z=\Delta \beta_{L} L_{M P C}+B_{\text {roundtrip }}=2 m \pi,
$$

where $B_{\text {roundrip }}$ is the B-integral per roundtrip that can be evaluated for a given gas-filled MPC configuration using [13]:

$$
B_{\text {roundtrip }}=\frac{8 \pi n_{2} P_{\text {peak }}}{\lambda_{p}{ }^{2}} \arctan \left(\sqrt{\frac{L}{2 R-L}}\right)
$$


The linear (corresponding to Eq. (6)) and nonlinear (Eq. (7)) FWM QPM curves for the experiment reported in [16] are plotted in Fig. 5.

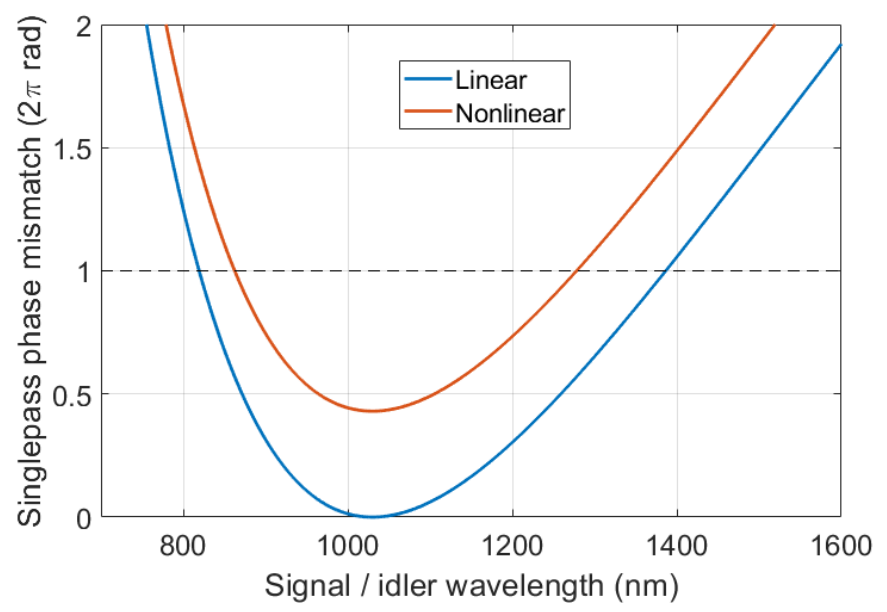

Fig. 5: Linear $\left(B_{\text {roundtrip }}=0\right)$ and nonlinear QPM curves for FWM corresponding to [16].

We first notice on the linear curve that, due to the normal dispersion regime, perfect phase matching is not possible elsewhere than at the pump wavelength, which corresponds to simple SPM. In the linear approximation, the first order QPM sidebands are located at $820 \mathrm{~nm}$ and $1385 \mathrm{~nm}$. The nonlinear contribution to phase matching shifts the curve upwards, resulting in phase-matched sidebands closer to the pump wavelength. Note that in the nonlinear QPM condition described in Eq. (7), it is assumed that SPM at the signal / idler wavelength is negligible, so that the curve is meaningless at wavelengths that overlap with the pump spectrum. In this region the interaction is always phase-matched since it corresponds to pure SPM.

We observe that nonlinear QPM of first order $(m=1)$ occurs at signal and idler wavelength around $860 \mathrm{~nm}$ and $1280 \mathrm{~nm}$, which further backs our hypothesis that QPM FWM is at the origin of the observed spectral content. The Rayleigh range in [16] is $10 \mathrm{~cm}$, while the average coherence length is greater than $1 \mathrm{~m}$, which verifies the condition for an efficient QPM process. The generated wavelengths in the simulation shown in Fig. 4 match very well those predicted by the nonlinear phase matching condition. We have verified that this holds true when changing the parameters such as peak power, MPC length, etc. The experimentally reported value of around $930 \mathrm{~nm}$ differs quite a bit, this might possibly due to uncertainties in the actual peak power, and the exact dispersion of argon. It could also be due to additional dispersion introduced at the mirror coatings that is not accounted for here.

The experiment described in [17] can be interpreted similarly with a quasi-phase matching that is satisfied at $895 \mathrm{~nm}$ and $1235 \mathrm{~nm}$, while the numerical simulation shows the initial appearance of sidebands at these wavelengths, with a progressive shift towards a greater wavelength separation from the pump upon propagation. We believe this shift to be due to the decrease of peak power caused by the non-negligible group-velocity dispersion of argon at a pressure of 4 bar.

It should be noted that the gain provided by this process is very high since what is observed is essentially optical parametric generation. Although a parasitic effect in the reported compression experiment, this process could be used, e. g. to amplify ultrashort pulses, as is already the case in optical fibers [26] or gas-filled capillaries [28,29]. This FWM phenomenon bears a lot of resemblance with modulation instability in dispersion oscillating fibers [30], for which a similar QPM condition exists, and therefore can be observed in normal dispersion regime as well. The main difference is that the oscillating quantity along the propagation distance in our case is the nonlinearity. 


\section{Conclusion}

We have presented a hybrid nonlinear pulse propagation model that is based on a rather complete approach in the time domain, and includes a basic description of Gaussian beam size and radius of curvature in the spatial domain. It is well suited to describe propagation in nonlinear MPCs, for which the spatial nonlinearity is often low enough to consider that the spatial profile remains Gaussian and negligible space-time-couplings are introduced. This approach allows to include the influence of the caustic evolution on the nonlinearity. The model is validated against a more complete but computation intensive numerical approach, as well as two recent experiments that are at the state of the art in terms of performances. Finally, it allows us to identify the origin of a parasitic process in these experiments, which we believe is due to QPM FWM. Although the model is only applied to rare gas-filled near-concentric MPCs in this work, it is straightforward to apply it in the case of molecular gases or MPCs including solid plates of medium, in arbitrary geometries, to describe a large panel of possible nonlinear interactions.

\section{Funding information}

Agence Nationale de la Recherche (ANR) (ANR-10-LABX-0039-PALM, ANR-16-CE300027-01-HELLIX, ANR-19-CE30-0001-02-MIRTHYX).

\section{Disclosures}

The authors declare no conflicts of interest.

\section{References}

1. G. P. Agrawal, "Nonlinear fiber optics," 5th edition, Academic Press (2012).

2. A. Couairon, E. Brambilla, T. Corti, D. Majus, O. de J. Ramırez-Gongora4, and M. Kolesik, "Practitioner's guide to laser pulse propagation models and simulation", Eur. Phys. J. Special Topics 199, 5-76 (2011).

3. John M. Dudley, Goëry Genty, and Stéphane Coen, "Supercontinuum generation in photonic crystal fiber," Rev. Mod. Phys. 78, 1135-1184 (2006).

4. A. Couairon, A. Mysyrowicz, "Femtosecond filamentation in transparent media," Phys. Reports 441, 47-189 (2007).

5. A. Dubietis, A. Couairon "Ultrafast supercontinuum generation in transparent solid-state media," Springer (2019).

6. Julien Pouysegur, Martin Delaigue, Yoann Zaouter, Clemens Hönninger, Eric Mottay, Anaël Jaffrès, Pascal Loiseau, Bruno Viana, Patrick Georges, and Frédéric Druon, "Sub-100-fs Yb:CALGO nonlinear regenerative amplifier," Opt. Lett. 38, 5180-5183 (2013).

7. Jan Schulte, Thomas Sartorius, Johannes Weitenberg, Andreas Vernaleken, and Peter Russbueldt, "Nonlinear pulse compression in a multi-pass cell," Opt. Lett. 41, 4511-4514 (2016).

8. M. Ueffing, S. Reiger, M. Kaumanns, V. Pervak, M. Trubetskov, T. Nubbemeyer, and F. Krausz, "Nonlinear pulse compression in a gas-filled multipass cell," Opt. Lett. 43, 2070-2073 (2018).

9. L. Lavenu, M. Natile, F. Guichard, Y. Zaouter, X. Delen, M. Hanna, E. Mottay, and P. Georges, "Nonlinear pulse compression based on a gas-filled multipass cell," Opt. Lett. 43, 2252-2255 (2018).

10. L. Lavenu, M. Natile, F. Guichard, X. Délen, M. Hanna, Y. Zaouter, and P. Georges, "High-power two-cycle ultrafast source based on hybrid nonlinear compression," Opt. Express 27, 1958-1967 (2019).

11. Nour Daher, Florent Guichard, Spencer W. Jolly, Xavier Délen, Fabien Quéré, Marc Hanna, and Patrick Georges, "Multipass cells: 1D numerical model and investigation of spatio-spectral couplings at high nonlinearity," J. Opt. Soc. Am. B 37, 993-999 (2020).

12. Patrick L. Kramer, Matthew Windeler, Katalin Mecseki, Elio G. Champenois, Matthias C. Hoffmann, Franz Tavella, "Enabling high repetition rate nonlinear THz science with a kilowatt-class sub-100 fs laser source," arXiv:2002.05286 [physics.optics].

13. M. Hanna, X. Délen, L. Lavenu, F. Guichard, Y. Zaouter, F. Druon, and P. Georges, "Nonlinear temporal compression in multipass cells: theory," J. Opt. Soc. Am. B 34, 1340-1347 (2017).

14. A. G. Kostenbauder, "Ray-Pulse Matrices: A Rational Treatment for Dispersive Optical Systems," IEEE J. Quant. Elect. 261148 - 1157 (1990).

15. C. Jirauschek and F. X. Kärtner, "Gaussian pulse dynamics in gain media with Kerr nonlinearity,” J. Opt. Soc. Am. B 23, 1776-1784 (2006).

16. M. Kaumanns, V. Pervak, D. Kormin, V. Leshchenko, A. Kessel, M. Ueffing, Y. Chen, and T. Nubbemeyer, "Multipass spectral broadening of $18 \mathrm{~mJ}$ pulses compressible from $1.3 \mathrm{ps}$ to $41 \mathrm{fs}$," Opt. Lett. 43, 5877-5880 (2018).

17. P. Russbueldt, J. Weitenberg, J. Schulte, R. Meyer, C. Meinhardt, H. D. Hoffmann, and R. Poprawe, "Scalable 30 fs laser source with 530 W average power," Opt. Lett. 44, 5222-5225 (2019). 
18. A. E. Siegman, "Lasers,” University Science Books (1986).

19. S. Leghmizi, A. Hasnaoui, B. Boubaha, A. Aissani and K. Ait-Ameur, "On the different ways for defining the effective focal length of a Kerr lens effect," Laser Phys. 27, 106201 (2017).

20. Gadi Fibich and Alexander L. Gaeta, "Critical power for self-focusing in bulk media and in hollow waveguides," Opt. Lett. 25, 335-337 (2000).

21. W. Koechner, "Solid-state laser engineering," 6th edition, Springer, 2006.

22. T. Brabec and F. Krausz, "Nonlinear optical pulse propagation in the single-cycle regime", Phys. Rev. Lett. 78.3282 (1997).

23. M. E. Marhic, P. A. Andrekson, P. Petropoulos, S. Radic, C. Peucheret, and M. Jazayerifar, "Fiber optical parametric amplifiers in optical communication systems," Laser Photon Rev. 9, 50-74 (2015).

24. Marc Hanna, Frédéric Druon, and Patrick Georges, "Fiber optical parametric chirped-pulse amplification in the femtosecond regime," Opt. Express 14, 2783-2790 (2006).

25. Cesar Jauregui, Alexander Steinmetz, Jens Limpert, and Andreas Tünnermann, "High-power efficient generation of visible and mid-infrared radiation exploiting four-wave-mixing in optical fibers," Opt. Express 20, 24957-24965 (2012).

26. Walter Fu and Frank W. Wise, "Normal-dispersion fiber optical parametric chirped-pulse amplification," Opt. Lett. 43, 5331-5334 (2018).

27. J. A. Armstrong, N Bloembergen, J. Ducuing, and S. Pershan, "Interactions between light waves in a nonlinear dielectric", Phys. Rev. 127, 1918 (1962).

28. Charles G. Durfee, Sterling Backus, Henry C. Kapteyn, and Margaret M. Murnane, "Intense 8-fs pulse generation in the deep ultraviolet," Opt. Lett. 24, 697-699 (1999).

29. Federico Belli, Amir Abdolvand, John C. Travers, and Philip St. J. Russell, "Highly efficient deep UV generation by four-wave mixing in gas-filled hollow-core photonic crystal fiber," Opt. Lett. 44, 5509-5512 (2019).

30. Arnaud Mussot, Matteo Conforti, Stefano Trillo, Francois Copie, and Alexandre Kudlinski, "Modulation instability in dispersion oscillating fibers," Adv. Opt. Photon. 10, 1-42 (2018). 